УДК 539.26/27

DOI: $10.17223 / 24135542 / 17 / 1$

\title{
А.Н. Хархардин
}

Белгородский государственный технологический университет им. В.Г. Шухова (г. Белгород, Россия)

\section{Гибридизация атомных орбиталей при нагревании металлов}

\begin{abstract}
Получены формуль для расчета коэффициентов температурного расширения металлов, координационного числа и коэффициента взаимодействия атомов металлов. Метод расчета основан на развитии концепции о гибридизации внутренних s-электронов с р-электронами с образованием гибридных s-n-p-орбиталей при нагревании металлов. Гибридизачия орбиталей происходит в результате перетекания s-электронов на вакантную р-орбиталь с расширением и образованием энергетических зон их перекрывания согласно допустимым уровням энергии, правилам Клечковского, Хунда и приниипу Паули.
\end{abstract}

Ключевые слова: металлы, атомные орбитали, гибридизаиия.

\section{Введение}

Строение электронных оболочек, т.е. распределение электронов по орбитам элементов Периодической системы Менделеева, относится к холодным изолированным атомам, и поэтому оно является основным их состоянием. Построение заселенности орбит электронами обосновано квантовой теорией спектроскопических данных элементов, нагреваемых до плазменного состояния, с учетом заряда ядра атомов, четырех квантовых чисел $(n, l, m$ и $s)$, принципа Паули и правил Клечковского и Хунда [1]. Распределение электронов на внешних орбитах может быть совершенно разным, когда атомы элемента находятся в кристаллическом теле, в расплаве или в растворе. Так, при нагревании металлов энергетические зоны валентных электронов и зоны дозволенных правилом отбора близлежащих вакантных $p$-орбиталей расширяются в область разрешенных значений энергии с взаимным перекрыванием орбиталей перетекаемых на них $s$-электронов. При этом с увеличением области перекрывания в результате перетекания электронной плотности на вакантную $p$-орбиталь, частично или полностью заполненную переселенными при нагревании атома электронами, доля валентной электронной плотности на вакантной орбитали повышается и становится больше, чем число электронов на ней. Внутренние, переселенные $s$-электроны и электроны на $p$-орбите в результате перекрывания их электронных орбиталей образуют гибридные орбитали повышенной сферичности атома.

Одно из основных положений классической теории металлов гласит, что в кристаллической решетке металлов валентные электроны делокали- 
зованы к виду электронного газа и компенсируют силы отталкивания между положительно заряженными ионами. Для большинства типичных металлов действует правило высокотемпературного полиморфизма: вблизи точки плавления плотность упаковки атомов снижается и стремится к величине произвольной их упаковки, при этом протекает полиморфное превращение в объемноцентрированную (ОЦК) укладку атомов в кристаллической решетке. Полиморфное превращение от плотно упакованных гексагональной (ГПУ) или гранецентрированной (ГЦК) укладки атомов в кристаллической решетке к менее упакованной ОЦК происходит с увеличением эффективного радиуса взаимодействия атомов. Полиморфное превращение от ГПУ к ГЦК укладке атомов происходит с уменьшением радиуса атомов, а обратный переход - с увеличением их радиуса, что связано с изменением внутренней энергии металлов без изменения энтропии.

Внешними могут быть не только валентные электроны, но и внутренние $s$-электроны, переселенные нагреванием металла на $p$-орбиталь, суперпозиция (наложение) которых образует с преобладанием одной из них гибридные орбитали металлической связи между атомами. В результате бокового и (или) радиального перекрывания орбиталей соседних атомов в среде образующегося высвобождением определенной доли более свободного электронного газа формируются равновесная, компенсирующая сила отталкивания ядер и повышенная электронная плотность между ними по связям в кристаллической решетке. Электронная плотность в направлениях перекрывания этих орбиталей, т.е. в направлениях металлических связей между атомами, наибольшая в результате уплотнения перекрыванием и стягивающим (между собой ядра атомов) действием электронной компоненты.

Образование твердой, или кристаллической, фазы металлов с полной заселенностью электронами внешних $s$ - и $p$-орбит атомов происходит с учетом выполнения принципа плотной упаковки: в поле действия гравитационной силь укладка взаимодействующих элементов дискретности в жидкой фазе при ее затвердевании стремится к величине наиболее плотной - гексагональной их упаковке в твердой фазе. Прочность связи в структурах металлов зависит как от числа валентных электронов, которые может отдавать каждый атом в данной их координации, так и от степени блокирования заряда ядра в составе иона, т.е. от степени и типа перекрывания внешних связывающих орбиталей. Количество и направленность металлических связей в пространстве определяет координационное число атомов в кристаллической решетке металлов. Численная величина внешней гибридной орбитали и угловое распределение электронной плотности на сфере атома определяют тип кристаллического полиэдра и его координационное число.

Вычисление коэффициента температурного расширения, как и ряда других физических параметров металлов, с учетом только валентных (внешних) электронов не приводит к достоверным результатам. Определение величины этого показателя для металлов было получено эксперимен- 
тально [1, 2]. Согласно современным представлениям П.П. Кузьменко [1], металлическая связь в кристаллической решетке образуется в результате радиального перекрывания $p$-облаков неспаренных в атомах внешних электронов. По нашему мнению, особенность перекрывания электронных облаков металлической связи, обусловленной р-электронами, состоит в том, что наряду с радиальным перекрыванием орбиталей имеются и боковые их перекрывания.

\section{1. $s-n-p$ гибридизация атомных орбиталей}

Координационный многогранник ГПУ кристаллического полиэдра имеет симметричное и асимметричное расположение трех атомов верхнего и нижнего слоя, соприкасающихся с центральным атомом, который окружен шестью атомами среднего слоя. При этом по три $p$-орбитали симметрично располагаются вокруг верхней и нижней частей $p$-орбитали центрального атома с боковым перекрыванием согласно схеме

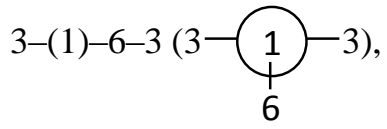

образуя гексагональную укладку атомов в кристаллической решетке без особых стереометрических затруднений. Здесь (1) - центральный атом в координационном полиэдре, а 12 атомов окружают центральный. Согласно современным представлениям, если с верхней и нижней частями р-облака симметрично перекрывается по три таких же облака с противоположным спином, то образуется ГПУ плотно упакованная решетка.

Такое расположение в пространстве атомов с одной $p$-орбиталью и одним неспаренным электроном на ней приводит к геометрическому расположению атомов на плоскости условно среднего слоя в координационном многограннике в виде шестиугольника. Подобное расположение атомов в пространстве предполагает смешанное - боковое и радиальное - перекрывание $p$-орбиталей при перетекании на них внутреннего $s$-электрона с образованием гибридных орбиталей вида $s-n-p$.

Атомы металлов с полностью заполненными $s$ - или $p$-орбиталями, как правило, образуют плотную ГПУ укладку по принципу наиплотнейшей упаковки. Координационный многогранник ГПУ с асимметричным расположением трех атомов верхнего и нижнего слоев согласно схеме расположения 1-5-(1)-5-1 имеет пентагональную укладку атомов в двух средних слоях, расположенных вокруг центрального атома, характерную укладке атомов в элементах живой природы. Один атом верхнего и один атом самого нижнего слоя в приведенной выше структуре имеют радиальное $p$-перекрывание с центральным атомом в координационном полиэдре.

Атомы металлов с одним неспаренным внешним $s$ - или $p$-электроном либо с одним внутренним $d$-электроном, как правило, образуют ОЦК или ГЦК кристаллическую решетку. Элементарная кристаллическая ячейка ГЦК кристаллической решетки не имеет центрального атома, но имеет две схемы 
расположения атомов. Схема одной из них - 5-4-5, в ней четыре «центральных» атома имеют радиальное $p$-перекрывание, пять атомов в каждой из пяти (фактически двух) граней имеют радиальное $p$-перекрывание, а все 10 поверхностных атомов имеют радиальное $p$ - и смешанное $s-n-p$ перекрывание. Вторая схема - 1-6-6-1, - где шесть атомов второго слоя (первый и четвертый слои имеют по одному атому) ассиметрично расположенных над шестью атомами третьего слоя. Все 8 поверхностных атомов размещаются в лунках между тремя «центральными» атомами с боковым p-перекрыванием. Три верхних «центральных» атома ассиметрично расположены над тремя такими же атомами нижнего слоя. Три поверхностных атома вместе с «центральным» (диагональные атомы в поверхности кристаллической ячейки) образуют в ней четыре фрагмента с радиальным $p$-перекрыванием орбиталей. Следовательно, все атомы в кристаллической ГЦК ячейке имеют в основном радиальное $s$-n- $p$ и смешанное (боковое) перекрывание.

Покажем далее образование плотноупакованных кристаллических решеток и координационных полиэдров металлов с радиальным и боковым перекрыванием $s$ - $n$ - $p$-орбиталей.

Наличие в атоме внутреннего $s$-электрона приводит к стереометрическому решению образования правильной ОЦК кристаллической решетки в результате образования $s-n-p$ гибридных орбиталей по схеме следующего вида:

$$
s^{1} p^{1} \rightarrow s^{\prime} \backslash_{p p^{1} \rightarrow s^{\prime}}{ }^{2} \backslash_{p} u s^{2} p^{1} \rightarrow s^{1} s^{I^{1}} \backslash_{p p^{1} \rightarrow s^{\prime}}{ }^{2} \backslash_{p p^{1}} .
$$

Парное и тройное расположение атомов с двумя $p$-орбиталями центрального атома приводит к стереометрическому затруднению образования соответствующих кристаллических решеток. Наличие в атоме внутренних $s$-электронов приводит к перетеканию их на вакантную $p$-орбиталь, взаимному отталкиванию $p$-орбиталей с нарушением ортогональности и расположению их вокруг центрального атома согласно координационному числу в кристаллической решетке в результате образования соответствующих гибридных орбиталей в зависимости от температуры металла по схеме следующего вида:

$$
\begin{aligned}
& s^{1} p^{2} \rightarrow s^{I^{1}} \backslash_{p p^{2} \rightarrow s^{\prime}} \backslash_{p p^{1} \rightarrow s^{\prime}} \backslash_{p,} \\
& s^{2} p^{2} \rightarrow s^{1} s^{1} \backslash_{p p^{2} \rightarrow s^{1} s^{\prime}}{ }^{2} \backslash_{p p^{1} \rightarrow s^{1} s} /^{3} \backslash_{p}
\end{aligned}
$$

Здесь имеют место тетраэдрическая и октаэдрическая направленность гибридных связей и смешанное перекрывание внешних $p$-орбиталей неспаренных и спаренных $p$-электронов. Наличие внутренних $s$-электронов в атоме приводит к перетеканию его плотности на вакантную $p$-орбиталь, к образованию в зависимости от температуры металла гибридных орбиталей с нарушением ортогональности внешних $p$-орбиталей при образовании металлической связи в соответствующих кристаллических решетках: 


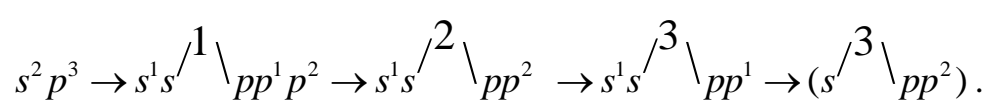

Здесь имеет место смешанное перекрывание внешних $p$-орбиталей с тетраэдрической и октаэдрической направленностью гибридных связей в группе атомов. При нагревании металла от абсолютного нуля до температуры кипения включительно происходит последовательное образование гибридных орбиталей такого вида, что число в них возбужденных внешних электронов также возрастает.

Преимущество сил притяжения над силами отталкивания между атомами при определенной температуре и в соответствии с образующейся при этом гибридной орбиталью металлической связи между ними приводит к взаимному полиморфизму - от ОЦК к ГЦК кристаллической решетке и наоборот. Элементарная кристаллическая ячейка ГЦК решетки в поле действия силы гравитации и металлической связи не имеет центрального атома. Связь между атомами в ГЦК решетке осуществляется тремя p-орбиталями у каждого атома, из которых одна с боковым, а две с радиальным перекрыванием.

Для выполнения правила целочисленного значения $m=Z /(3 p)$ необходимо наличие внутренней $s$-орбитали с целью получения гибридной орбитали типа $s^{1} s-1-p p^{2}$ и $s^{1} s-2-p p^{1}$. Фактически для ГПУ кристаллической peшетки находится по одной $s$ - и по три $p$-орбитали, распределенных под действием $s$-орбитали в пространстве вокруг центрального атома в соответствии со схемой 3- (1)6-3. Здесь $Z$ - координационное число координационного многогранника ГПУ атомов.

Так как металлы Периодической системы элементов в основном состоянии не имеют внешних $d$-орбиталей, но протяженность внутренних $d$-орбиталей выходит за сферы действия внешних валентных $s$ - и $p$-орбиталей, то вероятность образования $d$-орбиталями плотно упакованных структур в области низких температур до точки плавления наибольшая. В области высоких температур вероятность образования $d$-орбиталями высокотемпературных полиморфных структур с ГПУ и ГЦК укладкой в них атомов наименьшая. Такие структуры образуются в основном в результате $s-p$ - $d$-гибридизации внешних $s$-орбиталей и внутренних $d$-орбиталей, выходящих своей протяженностью за сферы действия $s$ - и $p$-орбиталей. Образуемые $s$ - $n$ - $p$ - и $s$ - $n$ - $d$-гибридные орбитали в плотно упакованных структурах имеют смешанный характер радиального и бокового перекрывания.

Так, в координационном многограннике гексагональной кристаллической решетки со схемой расположения атомов 3-(1)6-3 связь между ними осуществляется только тремя р-орбиталями у каждого атома (одна с радиальным и две с боковым перекрыванием) по схемам их фрагментов: $-1-1-1,2-1-2$ и 3-(1)-3, что соответствует $s^{1} s-1-p p^{2}, s^{1} s-2-p p^{1}$ и $s^{1} s-3-p-$ гибридным орбиталям. Распределение электронов на внешних орбитах $s^{1} p^{3}$ и $s^{2} p^{n}$ соответствует основному состоянию атомов металлоидных 
элементов. При этом для металлов с ГПУ кристаллической решеткой характерна полная заселенность электронами предвнешней и внешней электронных орбит в основном состоянии атома. Формирование высокотемпературных и высокоплотных полиморфных укладок атомов возможно с образованием $s-n-d$-гибридной металлической связи $d_{x y}-d_{y z}-d_{x}^{2}-y^{2}$ или $d_{x y}-$ $d_{y z}-d_{x}^{2}-z^{2}$ и тому подобных внешних орбиталей.

Приведем результаты образования связей перекрывания орбиталей в других известных кристаллических решетках элементов, получаемые при рассмотрении двух основных полиморфных модификаций их координационных многогранников.

Металлическая связь в тетрагональной кристаллической решетке $(Z=10)$ образуется в результате бокового перекрывания двух вдоль вертикальной оси $z$, горизонтальной оси $x$ и одной радиальной вдоль оси $y-$ $p$ - или $d z^{2}$ - орбиталей каждого атома по схеме 2-(1)6-2. Либо $p$-орбиталей вдоль осей $x$ и $y$ и радиального перекрывания одной $p$-орбитали вдоль оси $z$ по схеме $1-4(1) 4-1$, вид орбитали $-s^{1} s-2-p p^{1}$.

Металлическая связь в объемноцентрированной кристаллической решетке образуется в результате бокового перекрывания одной $p$-орбитали четырех атомов с верхней и нижней частями одной $p$-орбитали центрального атома вдоль оси $z$ и радиального перекрывания по одной $p$-орбитали атомов вдоль осей $x$ и $y\left(s-1-p p^{2}\right)$. Либо в результате радиальных перекрываний трех $p$-орбиталей в ее подрешетках $\left(s^{1} p^{3}\right)$ или двух $d_{x}^{2}-y^{2}-d_{x y}$, $d_{x}^{2}-y^{2}-d_{y z}$-орбиталей. Для данной кристаллической решетки возможен низкотемпературный и высокотемпературный полиморфизм (в частности, щелочных и других металлов) к гранецентрированной кристаллической решетке. При высоком давлении возможен полиморфизм в жидких металлах к плотноупакованным укладкам атомов, а в металлоидах он связан с разрывом химической (ковалентной) связи и ее перестройкой между атомами.

Химическая связь в тригональной кристаллической решетке с координационным числом $Z=8$ образуется в результате радиального перекрывания по одной $p$-орбитали вдоль оси $z$ и $y$ и бокового перекрывания одной $p$-орбитали вдоль оси $x\left(s^{1} s-1-p p^{2}\right)$ или радиальным перекрыванием двух $d_{x}^{2}-y^{2}-d_{x y}$ в плоскости среднего слоя, где $z=6$ и одной $d_{z}{ }^{2}$-орбитали вдоль оси $z$.

\section{2. Изменение информационной энтропии фазовых переходов первого рода}

Фазовые превращения первого рода, в том числе и полиморфные, протекают, как правило, с поглощением теплоты и, в зависимости от ее количества, подводимого к металлу в единицу времени, с определенной длительностью. Нагревание многих металлов сопровождается увеличением объема, характеризуемым коэффициентом температурного расширения, 
возбуждением электронов внешних орбиталей с расширением их энергетических уровней и увеличением зон их перекрывания с такими же орбиталями соседних атомов. Расширение энергетических уровней внешних, валентных орбиталей при нагревании металла до температуры кипения сопровождается последовательным переходом (перетеканием) электронной плотности предвнешних $s$-орбиталей на вакантную $p$-орбиталь с повышением их уровня энергии без нарушения правил Хунда. При этом происходят перестройка внешних $s$ - и $p$-орбиталей в гибридную орбиталь и заполнение $p$-орбиталей металлической связи неспаренными электронами. Совокупность электронной плотности валентных электронов, электронов вакантных орбиталей, энергетических зон их перекрывания и геометрическое радиального и бокового перекрывания внешних орбиталей образует металлическую связь.

Плавление металлов характеризуется разрушением металлической связи в кристаллической решетке в результате равенства или превышения кинетической энергии атомов над потенциальной энергией их взаимодействия, т.е. металлической связи в точке плавления. Разрушение монолита в точке плавления происходит не полностью, а на кластерные образования в плотной фазе флуктуации плотности. Дальнейшее нагревание металла до точки кипения происходит с уменьшением размера кластерных образований, в которых происходит дальнейшая перестройка металлической связи. При этом направленность металлической связи частично сглаживается возрастающей сферичностью внешней возбужденной электронной плотности, но остается еще достаточно выпуклой для образования высокого координационного числа в малых кластерах жидкой фазы. Прочность металлической связи в них возрастает в результате перестройки внешних орбиталей и пропорциональна температуре кипения металла. Число атомов в кластерных образованиях жидкой фазы и температура их разрушения пропорциональны образовавшемуся виду металлической связи, а размер кластеров пропорционален плотности упаковки и эффективному диаметру атомов в них. В точке кипения происходит вылет за поверхность жидкой фазы малых ассоциаций рыхлой фазы флуктуаций плотности, состоящей из трех и более атомов. Структура кластерных образований - ассоциаций атомов, полученных из парогазовой фазы после их охлаждения, - аморфизована. С увеличением размера таких микрочастиц степень кристалличности возрастает к их центру.

Плавление металлов, как и многих других веществ, характеризуется разрушением кристаллической решетки и уменьшением плотности упаковки атомов в жидкой фазе. Как следует из уравнения (1) при $\eta=0$ плотность их упаковки в исходной фазе должна быть не менее 0,4098. Но ряд веществ плавится с увеличением плотности упаковки атомов. Знание изменения плотности упаковки атомов при фазовых переходах необходимо в металлургических процессах, в порошковой промышленности изготовления изделий из металлов и сплавов. Для определения этого изменения - перепада плот- 
ности упаковки атомов при фазовых переходах - воспользуемся рекуррентной зависимостью для невзаимодействующих и слабовзаимодействующих элементов дискретности [3]:

$$
\eta=\eta_{1}\left[1 \pm \frac{1}{3 f \ln \left(120,754 \eta_{1}^{5}\right)}\right],
$$

где $\eta_{1}, \eta-$ плотность упаковки элементов дискретности в смежных фазах металлов в точке фазового превращения или полиморфизма, т.е. в более плотной и менее плотной фазе соответственно; $f$ - коэффициент взаимодействия атомов при данной температуре, который берется из потенциалов парного их взаимодействия (6-12, 3-9, 2-4), где $f \geq 1$ и $f \leq 1$.

Изменение информационной энтропии при топологических (фазовых) переходах кристаллического вещества к жидкой фазе, когда $\eta_{1}=0,6402894 \ldots 0,64658736 \ldots 0,64976$, a $f=1$ будет равно:

$$
\begin{gathered}
\Delta S=k_{Б} \ln \Delta \eta=k_{Б} \ln \left[\frac{\eta_{1}}{3 f \ln \left[2000(\sqrt{3}-1)^{9} \eta_{1}^{5}\right]}\right]= \\
=k_{Б} \ln \left\{\frac{0,640289 \ldots 0,64976}{3 f \ln \left[120,754(0,640289 \ldots 0,64976)^{5}\right]}\right\} \simeq-2,5 k_{Б} .
\end{gathered}
$$

где $k_{\text {Б }}$ - константа Больцмана.

При $\eta_{1}=0,6802 \ldots 0,7405$ из этого выражения получим: $\Delta S=k_{\text {Б }} \ln \Delta \eta=$ $=-(2,537 \ldots 2,590)$.

Коэффициент взаимодействия атомов $f$ выразим через отношение атомной массы А элемента к заряду ядра $z$ с учетом последовательного числа возбужденных (эффективных) внешних электронов $n_{e}$, изменяющегося от единицы до наибольшего их числа в металлической связи при данной температуре. Величина ее будет соответствовать числу топологических изменений при нагревании металла от абсолютного нуля до температуры кипения включительно. В этом отношении $\mathrm{A} / z$ введем поправку для учета сил притяжения и отталкивания атомов в виде обратных показателей степени в потенциале парного взаимодействия (6-12) Леннарда-Джонса:

$$
\Delta \eta= \pm \frac{\eta_{1}}{3\left\{\left(\frac{\mathrm{A}}{z}\right)^{n}-n_{e}\left[1 \pm\left(\frac{\mathrm{A}}{z}\right)^{1 / 6}-\left(\frac{\mathrm{A}}{z}\right)^{1 / 12}\right]\right\} \ln \left(120,754 \eta_{1}^{5}\right)},
$$

где $n_{e}-$ среднее число возбужденных внешних электронов на гибридной орбитали; $n$ - число электронов, соответствующее одноэлектронному $(n=1)$ или многоэлектронному приближению $(n \geq 2)$ в соответствии с требованием ряда превращений структуры, где наибольшее число электронов будет равно $(\mathrm{A} / z)^{2}-n_{e} \geq 1$. 
Первый знак «士» в уравнении (2) соответствует изменению плотности упаковки атомов в жидкой фазе в точке топологического превращения структуры, а второй знак «土» в скобках учитывает влияние сил притяжения или отталкивания соответственно. Знаменатель уравнения (2) определяет координационное число взаимодействующих атомов в твердой или жидкой фазе:

$$
\mathrm{Z}=3\left\{\left(\frac{\mathrm{A}}{\mathrm{Z}}\right)^{n}-n_{e}\left[1 \pm\left(\frac{\mathrm{A}}{\mathrm{Z}}\right)^{1 / 6}-\left(\frac{\mathrm{A}}{\mathrm{Z}}\right)^{1 / 12}\right]\right\} \ln \left(120,754 \eta_{1}^{5}\right) .
$$

Уравнения (2) и (3) позволяют, прежде всего по экспериментальным результатам изменения плотности упаковки атомов в смежных фазах в точке топологического превращения металлов, определить число $n_{e}$ возбужденных внешних электронов в атоме, задействованных в образовании металлической связи $\left(n_{e} \geq 1\right)$, и коэффициент взаимодействия атомов $f$ при данной температуре структурного изменения.

Для определения числа эффективных электронов на гибридных орбиталях, получаемого в результате расширения энергетических зон валентных электронов при нагревании металлов, введем следующие корректирующие дополнения. Для металлов с одним электроном на гибридной орбитали вида $s-1-p$ примем равным $n_{e}=1,5$, где среднее число $n_{e} \geq 1(1 \cdot 1,5)^{1 / 2} \geq$ $\geq 111,224745(1,225)$, для вида $s-2-p n_{\mathrm{e}}=2 \cdot 1,5=3$, среднее значение $n_{\mathrm{e}} \geq 1(2 \cdot 3)^{1 / 2} \geq 12,4495(2,45)$, для вида $s-3-p n_{\mathrm{e}}=4,5$, среднее значение величины $n_{\mathrm{e}} \geq 1(3 \cdot 4,5)^{1 / 2} \geq 13,6742(3,67)$. Кратное значение $n_{\mathrm{e}}=1,5$ получим из отношения числа вакантных $p$-орбиталей к числу $s$-электронов, переселенных при нагревании металла на более высокий энергетический уровень вакантной $p$-орбитали с расширением и перекрыванием электронных областей их энергетических уровней. Запишем выражение (3) для координационного числа $\mathrm{Z}$ в жидкой фазе в следующем виде:

$$
\begin{gathered}
\mathrm{Z}=3\left[\left(\frac{\mathrm{A}}{z}\right)^{n}-n_{e} f\right] \ln \left(120,754 \eta_{1}^{5}\right), \\
f=1 \pm\left(\frac{\mathrm{A}}{\mathrm{z}}\right)^{1 / 6}-\left(\frac{\mathrm{A}}{\mathrm{z}}\right)^{1 / 12} .
\end{gathered}
$$

Из выражения (4) общее число возбужденных внешних электронов в атоме при нагревании металла до температуры плавления примерно составит: $n_{e} \approx \mathrm{Z}_{\text {пр }} / \mathrm{Z}_{\text {кр }}$, где $\mathrm{Z}_{\text {пр }}, \mathrm{Z}_{\text {кр }}$ - координационное число атомов при произвольной их упаковке в жидкой фазе (2) и в кристаллической фазе соответственно. Тогда появляется возможность вычисления топологических свойств металлов для различных значений $\mathrm{Z}_{\text {пр}}$ :

$$
\Delta \eta \approx \pm \frac{\eta_{1}}{3\left[\left(\frac{\mathrm{A}}{z}\right)^{n}-\frac{Z_{\text {пр }}}{Z_{\text {кр }}} f\right] \ln \left(120,754 \eta_{1}^{5}\right)} .
$$


Для ОЦК кристаллической решетки $Z_{\text {Оцк }}=8$, а $Z_{\text {пр }}=8,6$, а для плотно упакованных решеток $Z_{\text {Гцк }}=12$, а $Z_{\text {пр }}=9,87$. При тепловом разрушении

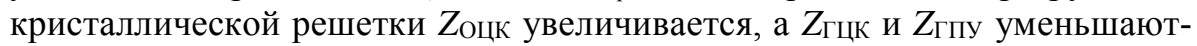
ся. Тогда число возбужденных внешних электронов в жидкой фазе при температуре кипения металла будет соответственно равно: $n_{e}=(\mathrm{A} / z)-1$ и $n_{e}=(\mathrm{A} / z)^{2}-1$. Из первого выражения, например при $\mathrm{A} / z=2$, имеем $n_{e}=1$, а при $\mathrm{A} / z=2,5$ получим $n_{e}=1,5$, что соответствует принятому выше допустимому его значению. Из второго выражения при $\mathrm{A} / z=2$ получим $n_{e}=2^{2}-1=3$, что предопределяет схему вычислений при температуре плавления металлов.

Приведем пример расчета топологических характеристик, например лития. Число возбужденных внешних электронов в атоме при нагревании лития до температуры кипения включительно $n_{e}=(6,941 / 3)^{2}-1=4,35$. Коэффициент парного взаимодействия атомов будет равен

$$
f=1 \pm(6,941 / 3)^{1 / 6}-(6,941 / 3)^{1 / 12}=1 \pm 0,07765 .
$$

Распределение внешних возбужденных электронов по орбитам в атоме при нагревании лития до температуры кипения включительно имеет вид:

$$
\mathrm{Li} \rightarrow 1 s^{2} 2 s^{1} \rightarrow 1 s^{2} 2 s^{\prime} \quad 2^{\backslash} p \rightarrow 1 s^{1} 2 s^{\prime} \quad 2^{\backslash} p 2 p^{1} \rightarrow 1 s^{1} 2 s^{\prime} \quad 2^{\backslash} p \rightarrow 2 s^{\prime}{ }^{\prime} 2^{\prime} p \ldots
$$

В скобках приведены наибольшие числа возбужденных внешних электронов с учетом $s$ - $n$ - $p$-гибридизации.

Изменение плотности упаковки атомов, связанное с увеличением их радиуса и температурным расширением металла при нагревании лития от абсолютного нуля до температур плавления и кипения, согласно уравнению (2) будет равно

$$
\Delta \eta=\frac{0,680175}{(6,941 / 3-f) 3 \ln \left(120,754 \cdot 0,680175^{5}\right)}=\frac{0,680175}{(2,3137-f) 8,600} .
$$

В области умеренных температур при $f=1-0,07765=0,92235$ из этого выражения получим: $\Delta \eta=0,0568$, в области повышенных температур при $f=1 \Delta \eta=0,0602$, в области пониженных температур при $f=1+0,07765$ имеем $\Delta \eta=0,0550$. Снижение плотности упаковки атомов в жидкой фазе вблизи температуры плавления - при температуре, характерной для всех металлов, когда плотность упаковки атомов снижается на величину отношения атомного объема металла при абсолютном нуле к атомному объему его при температуре, когда возможен переход к плотноупакованным структурам, соответствующей значению $n_{e}=1$ (при $\left.f=1 \ldots 0,92235\right)$, будет равна

$$
\begin{gathered}
\Delta \eta=\frac{0,6802}{\left(2,3137^{2}-1\right) 8,600}=0,01817, \\
\eta_{\mathrm{T}}=0,68017-0,01817=0,6620 .
\end{gathered}
$$


При $\eta_{1}=0,6620$ и $n_{e}=1,5 \ldots 2,5$ согласно уравнению (2) получим плотность произвольной упаковки атомов в жидкой фазе $\eta_{1}=0,6410 \ldots 0,6337$. Плотность упаковки атомов в твердой фазе вблизи температуры плавления при $\eta_{\text {оцк }}=0,6802, n_{e}=2,0$ и $f=1$ согласно уравнению (2) составит $\eta_{1}=0,6566$, тогда как плотность упаковки атомов в кластерных образованиях жидкой фазы при температуре плавления лития согласно уравнению (2) при $\eta_{\text {оцк }}=0,6802, n_{e}=2$ и $f>1$, при $n_{\mathrm{e}}=2,5$ и $f=1$ находится в пределах $\eta_{\text {кл }}=0,6554 \ldots 0,6525$.

Плотность упаковки атомов в жидкой фазе при температуре плавления лития находится в пределах

$$
\begin{gathered}
\Delta \eta=\frac{0,6802}{3\left(2,3137^{2}-2,5 \cdot 1,07765\right) 2,8667}=0,0297, \\
\eta_{ж}=0,6802-0,0297=0,6504 \\
\Delta \eta=\frac{0,6802}{3\left(2,3137^{2}-3 \cdot 0,92235\right) 2,8667}=0,0306, \\
\eta_{ж}=0,6802-0,0306=0,64959 .
\end{gathered}
$$

Наименьшая плотность упаковки атомов в кластерных образованиях жидкой фазы при температуре кипения лития будет равна

$$
\begin{gathered}
\Delta \eta=\frac{0,6525}{3\left(2,3137^{2}-4,5 \cdot 0,92235\right) 2,8667}=0,0631, \\
\eta_{\text {кл }}=0,6525-0,0631=0,5894 .
\end{gathered}
$$

Плотность упаковки атомов в жидкой фазе при температуре кипения лития находится в пределах

$$
\begin{gathered}
\Delta \eta=\frac{0,6504}{3\left(2,3137^{2}-4,5 \cdot 0,92235\right) 2,6431}=0,0682, \\
\eta_{ж}=0,6504-0,0682=0,5822, \\
\Delta \eta=\frac{0,64959}{3\left(2,3137^{2}-4,5 \cdot 0,92235\right) 2,6367}=0,0683, \\
\eta_{ж}=0,64959-0,0683=0,5813 .
\end{gathered}
$$

Координационное число атомов в жидкой фазе при температуре плавления лития будет равно

$$
Z=3\left(2,3137^{2}-4,5 \cdot 0,92235\right) 2,6367=9,51 .
$$

Следовательно, координационное число атомов в жидкой фазе лития описывается уравнением вида:

$$
Z=3 \cdot 1,2024 \ln \left(120,754 \eta_{1}^{5}\right) .
$$

Величина изменения плотности упаковки атомов при нагревании большинства металлов на один градус в интервале от абсолютного нуля до 
температуры плавления $\left(T_{\text {пл}}\right)$ является коэффициентом температурного объемного расширения $\alpha^{\prime}$. Температурный коэффициент объемного расширения обратно пропорционален температуре плавления, что вытекает из его дифференциальной формы: $\alpha^{\prime}=\frac{1}{V} \frac{d V}{d T}$, где $V$ - объем при абсолютном нуле температуры. Проинтегрируем это выражение в виде: $\int_{0}^{T_{n z}} \alpha^{\prime} d T=\int_{V}^{V_{n z}} \frac{d V}{V}$, получим

$$
\alpha^{\prime} T_{\text {пл }}=\ln \frac{V_{\text {пл }}}{V}=\Delta \eta,
$$

где $V_{\text {пл }}$ - объем твердой фазы металла вблизи температуры плавления.

Так как $\Delta \eta=\eta_{1} / 3 f \ln \left(120,754 \eta_{1}^{5}\right)$, то

$$
\alpha^{\prime}=\frac{\Delta \eta}{T_{\text {пл }}}=\frac{\eta_{1}}{3 f T_{\text {пл }} \ln \left(120,754 \eta_{1}^{5}\right)} .
$$

Поскольку коэффициент температурного объемного расширения

$$
\alpha^{\prime}=(1+\alpha)^{3}=1+3 \alpha^{2}+3 \alpha+3 \alpha^{3},
$$

то коэффициент температурного линейного расширения металлов $\alpha$ с незначительной погрешностью будет равен

$$
\alpha=\Delta \eta / 3 T_{\text {пл }}=\frac{\eta_{1}}{9 f T_{\text {пл }} \ln \left(120,754 \eta_{1}^{5}\right)} .
$$

Для металлов с ОЦК $\left(\eta_{1}=0,6802\right)$, с ГПУ и с ГЦК кристаллическими решетками $\left(\eta_{1}=0,74048\right)$ это уравнение упрощается соответственно к виду:

$$
\alpha=(0,0236 \ldots 0,025) / f T_{\text {пл }} .
$$

Коэффициент парного взаимодействия атомов в выражении $f \leq 1-$ для области низких и повышенных температур, $f \geq 1$ - для области повышенных температур и высокотемпературной области плавления металлов. Так, для некоторых металлов получим:

для осмия при $n=3 ;(4,5)$ и $f>1 \alpha=0,06 \ldots 0,068(0,06 \ldots 0,08) 10^{-5} \mathrm{~K}^{-1}$;

для иридия при $n=1$ и $f>1 \alpha=0,65(0,65) 10^{-5} \mathrm{~K}^{-1}$;

для родия при $n=1$ и $f \leq 1 \alpha=0,84 \ldots 0,87(0,85) 10^{-5} \mathrm{~K}^{-1}$;

для рутения при $n=1$ и $f=1 \alpha=0,991(0,991) 10^{-5} \mathrm{~K}^{-1}$;

для палладия при $n=1$ и $f \geq 1 \alpha=1,11 \ldots 1,37(1,25) 10^{-5} \mathrm{~K}^{-1}$;

для платины при $n=1$ и $f>1$, при $n=1,5$ и $f<1$

$\alpha=0,863 \ldots 1,08(0,892 \ldots 0,909) 10^{-5} \mathrm{~K}^{-1}$.

В скобках приведены экспериментальные справочные данные [2].

В таблице приведены сравнительные результаты расчета исследуемых характеристик некоторых металлов с экспериментальными справочными данными [2]. 


\section{Топологические свойства жидких и твердых металлов}

\begin{tabular}{|c|c|c|c|c|c|c|c|c|}
\hline \multirow[t]{2}{*}{$\begin{array}{l}\text { Эле- } \\
\text { мент }\end{array}$} & \multirow[t]{2}{*}{$\begin{array}{l}\text { Темпера- } \\
\text { тура плав- } \\
\text { ления, К }\end{array}$} & \multicolumn{2}{|c|}{$\begin{array}{c}\text { Плотность } \\
\text { упаковки атомов, } \\
\eta_{ж}\end{array}$} & \multirow{2}{*}{$\begin{array}{c}\text { Коэффициент } \\
\text { взаимодей- } \\
\text { ствия атомов, } \\
f\end{array}$} & \multicolumn{2}{|c|}{$\begin{array}{c}\text { Координационное } \\
\text { число, } Z\end{array}$} & \multicolumn{2}{|c|}{$\begin{array}{c}\text { Коэффициент } \\
\text { температурного } \\
\text { линейного рас- } \\
\text { ширения, } \\
1 \cdot 10^{5} \mathrm{~K}^{-1} \cdot \alpha\end{array}$} \\
\hline & & Расчет & $\begin{array}{l}\text { Экспе- } \\
\text { римент }\end{array}$ & & $\begin{array}{l}\text { Pac- } \\
\text { чет }\end{array}$ & $\begin{array}{c}\text { Экспери- } \\
\text { мент }\end{array}$ & \begin{tabular}{|c|} 
Рac- \\
чет
\end{tabular} & $\begin{array}{c}\text { Экспери- } \\
\text { мент }\end{array}$ \\
\hline $\mathrm{Li}$ & 452 & 0,650 & 0,65 & 1,2024 & 9,5 & 9,5 & $\begin{array}{c}4,44 / \\
5,83\end{array}$ & {$[4,72]$} \\
\hline $\mathrm{Na}$ & 371 & 0,645 & 0,69 & 1,6590 & 9,1 & 9,0 & $\begin{array}{c}6,95 / \\
7,11\end{array}$ & 7,2 \\
\hline K & 336 & 0,625 & 0,68 & 1,2345 & 9,0 & 9,0 & $\begin{array}{l}7,9 / \\
8,47\end{array}$ & 8,4 \\
\hline $\mathrm{Rb}$ & 312 & 0,635 & - & 1,1845 & 9,5 & 9,5 & $\begin{array}{c}8,41 / \\
9,1\end{array}$ & 9,0 \\
\hline Cs & 302 & 0,621 & 0,63 & 1,2500 & 9,0 & 9,0 & $\begin{array}{l}8,4 / \\
9,53\end{array}$ & - \\
\hline $\mathrm{Cu}$ & 1356 & 0,672 & 0,67 & 1,3020 & 11 & 10,9 & $\begin{array}{c}1,65 / \\
1,70\end{array}$ & 1,70 \\
\hline $\mathrm{Ag}$ & 1234 & 0,686 & 0,69 & 1,2673 & 11 & 11 & $\begin{array}{c}1,66 / \\
1,86\end{array}$ & 1,88 \\
\hline $\mathrm{Au}$ & 1336 & 0,682 & 0,68 & 1,2165 & 10,5 & 10,5 & $\begin{array}{c}1,33 / \\
1,42\end{array}$ & 1,42 \\
\hline $\mathrm{Be}$ & 1558 & 0,624 & - & 1,3760 & 10 & - & $\begin{array}{c}1,28 / \\
1,36\end{array}$ & 1,31 \\
\hline $\mathrm{Mg}$ & 923 & 0,669 & - & 1,2950 & 10,8 & 10,8 & $\begin{array}{c}2,48 / \\
2,64\end{array} \mid$ & 2,60 \\
\hline $\mathrm{Ca}$ & 1123 & 0,611 & - & 1,3580 & 9,5 & - & $\begin{array}{c}2,09 / \\
2,22\end{array} \mid$ & 2,20 \\
\hline $\mathrm{Zn}$ & 692 & 0,644 & 0,61 & 1,2495 & 9,71 & 9,7 & $\begin{array}{c}2,89 / \\
3,06\end{array}$ & 2,84 \\
\hline B & 2573 & 0,637 & - & 1,4219 & 10,8 & - & 0,83 & 0,83 \\
\hline $\mathrm{Al}$ & 933 & 0,671 & 0,65 & 1,3080 & 11 & 11 & $\begin{array}{c}2,34 / \\
2,49\end{array}$ & 2,46 \\
\hline $\mathrm{Pb}$ & 600 & 0,633 & 0,65 & 1,3849 & 10,4 & 10,4 & $\begin{array}{c}2,73 / \\
2,89\end{array}$ & 2,75 \\
\hline $\mathrm{Fe}$ & 1812 & 0,651 & - & 1,3591 & 10,8 & 10,8 & $\begin{array}{c}1,13 / \\
1,20\end{array}$ & 1,17 \\
\hline Co & 1767 & 0,626 & - & 1,264 & 9,3 & 9,3 & 1,27 & 1,34 \\
\hline $\mathrm{Ni}$ & 1728 & 0,640 & - & 1,3950 & 10,7 & 10,6 & $\begin{array}{c}1,32 / \\
1,40\end{array}$ & 1,35 \\
\hline Os & 3300 & $|0,645|$ & - & 1,3773 & 11,4 & - & 0,061 & 0,061 \\
\hline
\end{tabular}

Как видно из таблицы, величина расчетного коэффициента температурного линейного расширения большинства металлов меньше экспериментальной, что связано с определением его вблизи температуры плавления. Экспериментальная величина его определяется обычно в интервале температур несколько ниже температуры плавления металла. 


\section{Заключение}

Таким образом, принятое кратное значение числа возбужденных внешних электронов $3 / 2$ на гибридных орбиталях атомов и коэффициент их парного взаимодействия, полученный из потенциала Леннарда-Джонса, достаточно оправданы совпадающими результатами расчета показателей топологии жидких и твердых металлов с экспериментальными данными. Кратные значения чисел возбужденных электронов 1,5, 2, 2,5, 3 являются постоянными Грюнайзена для разных металлов. Экспериментальная плотность упаковки атомов в жидких металлах, полученная методом радиальной функции распределения плотности, видимо, относится к кластерным образованиям в жидкой фазе.

При нагревании металлов в образовании гибридных орбиталей в атоме принимают участие $s$-электроны, переселенные из внутренних орбит на вакантную $p$-орбиталь согласно правилам Хунда и Клечковского. Искаженная объемноцентрированная укладка атомов в кластерных образованиях жидкой фазы вблизи температуры плавления металлов формируется наличием в атоме внутренних $s$-электронов, переселенных на внешнюю $p$-орбиталь с образованием s-n-p-гибридных орбиталей с боковым перекрыванием $p$-электронов восьми атомов в системе с центральным атомом и с радиальным перекрыванием $p$-орбиталей в ее подрешетках. Гексагональная, гранецентрированная и тетрагональная укладки атомов в кристаллических решетках образуются в результате смешанного (бокового и радиального) перекрывания $s-n-p-, s-n-d$-электронов, образуя гибридные орбитали при наличии внутренних $s$-электронов. Искаженные их структуры в кластерных образованиях жидкой фазы вблизи температуры плавления металлов характеризуются переходом (переселением) внутренних $s$-электронов на гибридные $s$-n-p-орбитали по правилам Хунда и Кличковского, где $n \geq 1$.

Образование гибридных орбиталей вблизи температуры плавления металлов приводит к перестройке плотно упакованных (гексагональной и гранецентрированной) структур к искаженной тетрагональной и далее к объемноцентрированной укладке атомов или к жидкофазному полиморфизму с последующим разрушением при нагревании полиморфных структур до кластерных образований в жидкой фазе вплоть до температуры кипения. При охлаждении металлов с приближением к абсолютному нулю температуры низкотемпературный полиморфизм приводит в конечном итоге к гексагональной укладке атомов. Как показывают расчеты, атомы некоторых металлов, в частности с объемноцентрированной укладкой, при комнатной температуре характеризуются наличием гибридных орбиталей.

Знание порядка гибридных орбиталей при различной температуре позволит определить соответствующие им характерные температурные величины и точки изменения физических свойств металлов, топологию уровней энергии в атоме, величину и природу металлической связи, электрои теплопроводности металлов и объяснить многие физические свойства 
и эффекты в жидких и твердых металлах. Так, быстрое охлаждение перегретой жидкой фазы с локальными образованиями гибридных орбиталей может привести к мгновенному их разрушению с выделением теплоты и, как следствие, к вспучиванию жидкого металла.

Данный метод расчета может быть использован для определения координационного числа атомов и плотности их упаковки в жидкой фазе и в кластерных образованиях. Предлагаемый расчетный метод определения коэффициентов температурного объемного и линейного расширения металлов может быть использован и для металлических сплавов.

Статья подготовлена в рамках программь развития опорного университета на базе БГТУ им. В.Г. Шухова

\section{Литература}

1. Андреев С.Н.; Смирнова М.Ф. Строение электронных оболочек атомов. Теория химической связи. Л. : Изд-во Ленингр. гос. ун-та, 1974. 70 с.

2. Скрышевский А.Ф. Структурный анализ жидкостей и аморфных тел. М. : Высш. школа, 1980. 328 с.

3. Хархардин А.Н. Дискретная топология. Белгород : Изд-во БГТУ, 2016. 620 с.

\section{Информация об авторе:}

Хархардин Анатолий Николаевич, профессор, доктор технических наук, профессор кафедры материаловедения и технологии материалов Белгородского государственного технологического университета им. В.Г. Шухова (Белгород, Россия). E-mail: graf38@ bk.ru

Tomsk State University Journal of Chemistry, 2020, 17, 6-21. DOI: 10.17223/24135542/17/1

\section{A.N. Kharkhardin}

Belgorod State Technological University named after V.G. Shukhov (Belgorod, Russia)

\section{Hybridization of atomic orbitals when heated metal}

The method of calculation of coefficients of temperature expansion of metals, coordination number and coefficient of interaction of atoms of metals is developed. The calculation method is based on the development of the concept of hybridization of internal s-electrons with p-electrons to form hybrid s- $n$-p-orbitals when metals are heated. The hybridization of orbitals occurs as a result of the blend of s-electrons to the vacant p-orbital with formation of energy bands according to their overlap with the permissible levels of energy, Kleczkowska rules, Hund and Pauli principle.

Keywords: metals, atomic orbitals, hybridization.

\section{References}

1. Andreev, S.N.; Smirnov, M.F. Structure of electron shells of atoms. Theory of chemical bonding; Leningrad University Publ.: Leningrad, 1974; p. 70. 
2. Skrisevskiy A.F. Structural analysis of liquids and amorphous bodies. M.: Higher school, 1980, 167-185.

3. KharkhardIn A.N. Discrete topology. Belgorod: Ed.-in BSTU, 2016, 620.

\section{Information about the author:}

Kharkhardin Anatolij, professor, doctor of Science (Technical field), professor at Department "Materials sciences and materials technology", Belgorod State Technological University named after V.G. Shukhov (Belgorod, Russia).E-mail: graf38@bk.ru 